\title{
Chapitre 8 La simulation numérique des accidents de fusion du cœur
}

\subsection{Logiciels intégraux et logiciels mécanistes}

Des logiciels de simulation numérique des accidents de fusion du cœur ont été développés aux États-Unis à partir des années 1970, en support aux études probabilistes telles que celles présentées dans le rapport américain WASH 1400 [1]. Ensuite, l'accident de TMI-2 aux États-Unis en 1979 a conduit dans ce pays à améliorer la simulation de ces accidents et à développer des études probabilistes de sûreté (EPS) de niveau 2. Après le rapport WASH 1400, un second rapport important présente une telle étude probabiliste pour cinq réacteurs des États-Unis [2]. Puis, à la fin des années 1980, l’Europe et le Japon ont décidé de développer leurs propres logiciels de simulation des accidents de fusion du cœur et leurs propres études probabilistes. À cette même époque, ces logiciels ont progressivement été utilisés pour élaborer ou apprécier des mesures de prévention ou de limitation des conséquences des accidents de fusion du cœur, ainsi que pour la formation des opérateurs de réacteurs.

Deux types de logiciels ont été développés :

- des logiciels intégraux (ou systèmes de logiciels) simulant l'ensemble d'un accident avec fusion du cœur, depuis l'événement initiateur jusqu'aux rejets éventuels de radionucléides à l'extérieur de l'enceinte de confinement ;

- des logiciels détaillés ou mécanistes simulant plus finement les phénomènes intervenant au cours d'une partie de l'accident, par exemple la dégradation du cœur, le relâchement des produits de fission ou la combustion de l'hydrogène dans l'enceinte. 


\subsubsection{Logiciels intégraux}

Dans les années 1980, après l'accident de TMI-2, deux logiciels intégraux ont été développés en parallèle aux États-Unis: MAAP pour les électriciens nucléaires et MELCOR pour les autorités réglementaires. À la fin des années 1980, le Japon et la France ont démarré le développement de logiciels analogues, respectivement THALES [2] pour JAERI [3] et ESCADRE pour l'IRSN [4]. Puis l'IRSN a décidé de lancer avec la GRS le développement commun d'un logiciel dénommé ASTEC, destiné à remplacer ESCADRE. Au Japon, un ambitieux projet a démarré à NUPEC avec le système SAMPSON de logiciels détaillés [5] mais, compte tenu de la décroissance de la R\&D sur les accidents de fusion du cœur au Japon, les développements correspondants ont quasiment été arrêtés vers 2001. L'organisme japonais JAEA (successeur de JAERI) vient de redémarrer le développement du logiciel THALES. Au début des années 2000, la Russie a entamé le développement du système de logiciels SOCRAT [6].

Il existe cinq logiciels intégraux dans le monde : MAAP, MELCOR, ASTEC, SAMPSON et SOCRAT (ne sont pas présentés ici certains logiciels spécifiques qui simulent les accidents de fusion du cœur dans les réacteurs CANDU à eau lourde de conception canadienne, par exemple le logiciel ISAAC développé par KAERI en Corée du Sud) mais trois seulement sont largement diffusés et utilisés :

- ASTEC, co-développé par l'IRSN et son homologue allemand, la GRS ;

- MAAP4, développé par la société Fauske \& Associates, Inc. (FAI) aux États-Unis ;

- MELCOR, développé par Sandia National Laboratories (SNL) aux États-Unis pour l'US NRC (US Nuclear Regulatory Commission).

Un logiciel intégral doit répondre aux exigences suivantes :

- traiter de façon exhaustive les phénomènes physiques intervenant lors d'un accident de fusion du cœur ;

- simuler le comportement des principaux systèmes de sûreté du réacteur ;

- traiter complètement les couplages entre phénomènes, par exemple la décroissance de la puissance résiduelle en fonction des relâchements de produits de fission (PF) depuis le combustible, l'oxydation des métaux en fonction de la quantité d'oxygène ou de vapeur d'eau disponible ou le refroidissement du corium dans le puits de cuve, en cours d'interaction corium-béton, par rayonnement et par convection dans l'enceinte de confinement ;

- être modulaire, pour faciliter notamment les comparaisons avec des résultats expérimentaux ;

- être rapide afin de pouvoir réaliser un grand nombre de calculs de scénarios pour les EPS2 dans un délai raisonnable. Il faut pour cela que le temps de calcul soit inférieur au temps réel de l'accident.

Les logiciels intégraux sont utilisés pour des études de sûreté des réacteurs, notamment pour l'évaluation des rejets envisageables, pour des EPS de niveau 2, ainsi que pour 
des études menées en préparation à la gestion d'un éventuel accident de fusion du cœur en vue de définir des guides d'intervention en accident grave. Ils peuvent être également utilisés pour la vérification du dimensionnement de dispositifs tels que les vannes de décharge du pressuriseur ou les recombineurs dans l'enceinte de confinement.

Les principaux domaines physiques couverts sont les suivants :

- la thermohydraulique des circuits primaire et secondaire au début de l'accident (" tête de séquence ») ;

- la fusion du cœur, avec le relâchement des produits de fission depuis les crayons combustibles et les relâchements d'aérosols depuis les structures présentes dans la cuve (barres de commande, grilles, etc.) ;

- la relocalisation du corium dans le fond de la cuve et la défaillance de celle-ci, entraînant la coulée de corium dans le puits de cuve ;

- l'interaction corium-béton du radier du puits de cuve ;

- le transport et le dépôt des produits de fission et des aérosols dans les circuits primaire et secondaire ;

- la thermohydraulique dans l'enceinte de confinement ;

- le transport et le dépôt des produits de fission et des aérosols dans l'enceinte de confinement ;

- la chimie des produits de fission, en particulier l'iode et le ruthénium, dans l'enceinte de confinement et leur rejet éventuel dans l'environnement.

En règle générale, les logiciels intégraux ne traitent ni l'explosion de vapeur ni la tenue mécanique de l'enceinte, qui sont traitées par des logiciels détaillés.

Les accidents de fusion du cœur comportent des phénomènes transitoires dans des milieux solides, liquides ou gazeux, qui échangent masses, quantités de mouvement et énergies. Un logiciel intégral doit coupler tous ces phénomènes qui interviennent à des échelles différentes dans l'espace et dans le temps, par exemple des phénomènes qui durent quelques secondes comme l'échauffement direct de l'enceinte ou quelques heures comme la montée en pression de l'enceinte de confinement sous l'effet des relâchements de gaz lors de l'interaction corium-béton, ou bien des réactions chimiques localisées entre gaine et combustible mais aussi des écoulements de vapeur sur toute la hauteur du cœur. Ces logiciels font ainsi naturellement appel à différentes disciplines scientifiques : thermique, thermohydraulique, mécanique des structures, chimie, etc.

Les équations de conservation de la masse et de l'énergie interne sont résolues, pour chacun des constituants présents, dans des volumes (nommés « volumes de contrôle ») qui définissent un maillage de l'espace. Les circuits primaire et secondaire et l'enceinte de confinement peuvent être représentés par un nombre quelconque de volumes (en général entre 10 et 50 pour l'enceinte) ${ }^{1}$. Le cœur du réacteur est maillé

1. Excepté pour le logiciel MAAP4 où le nombre maximal est fixé à 14 pour le circuit primaire et deux pour le circuit secondaire. 
en $2 \mathrm{D}$ axisymétrique : en général quatre à sept couronnes radiales et 10 à 30 volumes axiaux. Le corium durant l'interaction corium-béton est discrétisé en un, deux ou trois volumes, représentant des couches moyennées (métaux d'une part, oxydes lourds et oxydes légers provenant de la décomposition du béton d'autre part).

Le degré de finesse choisi pour la discrétisation en volumes de contrôle influe considérablement sur le temps de calcul. Il est ainsi possible de descendre à une ou deux heures de calcul par jour d'accident en adoptant les nombres minimaux de volumes indiqués ci-dessus, mais avec le risque de réduire la fiabilité des résultats.

Les modèles thermohydrauliques jouent un rôle fondamental dans les logiciels intégraux car ils sont étroitement imbriqués aux autres modèles : en général chaque volume de contrôle contient deux zones de températures différentes, une zone fluide (simple phase liquide ou double phase en ébullition) et une zone gazeuse qui peut contenir un brouillard d'eau. Ces volumes peuvent être entourés de structures solides qui conduisent la chaleur. Ils sont connectés par des jonctions. Les vitesses des fluides circulant dans ces jonctions sont calculées en résolvant les équations de quantité de mouvement des fluides : la vitesse moyenne du fluide est calculée en régime transitoire à l'aide de l'équation de Bernoulli généralisée avec prise en compte des pertes de charge ; la différence de vitesse entre le liquide et le gaz est alors évaluée à partir de cette vitesse moyenne à l'aide d'une corrélation dite de "glissement entre phases ». Dans le logiciel ASTEC, pour les circuits primaire et secondaire, une modélisation plus complexe de volumes 1D (de type " tuyau ») contenant un mélange diphasique est utilisée. Les modélisations thermohydrauliques des circuits primaire et secondaire et de l'enceinte sont étroitement couplées par la modélisation des échanges thermiques entre les circuits et l'enceinte de confinement ou par la décharge d'eau ou de vapeur des circuits vers l'enceinte en cas de rupture de tuyauterie. Cela permet de modéliser de manière simple certains automatismes d'un réacteur, comme le démarrage de certains systèmes (injection de sécurité, aspersion dans l'enceinte) sur des seuils de pression élevée dans l'enceinte.

Les logiciels intégraux sont de grande taille : de 400000 à 500000 instructions et 1000 à 1500 sous-programmes pour les logiciels ASTEC et MELCOR, environ 350000 instructions et 700 sous-programmes pour le logiciel MAAP. Ils sont structurés en modules qui correspondent approximativement aux principales zones du réacteur. Ces modules sont liés par un programme informatique qui gère l'évolution des pas de temps du schéma de résolution numérique, et les échanges de données et assure la conservation des bilans de masse et d'énergie. Le langage de programmation est le Fortran 95 ou ses successeurs.

Une fonctionnalité essentielle de ces logiciels est d'offrir à l'utilisateur la possibilité de définir les hypothèses de l'accident étudié de la manière la plus flexible et la plus simple possible, souvent à l'aide d'un langage de commande spécifique ou à l'aide d'une Interface-Homme-Machine. Il est ainsi possible de simuler les systèmes de sûreté intervenant au cours de l'accident, ainsi que les procédures mises en œuvre par les opérateurs pour la « gestion » de l'accident :

- pour les circuits: dépressurisation volontaire, envoi d'eau sur le cœur dégradé dans la cuve, etc. ; 
- pour l'enceinte de confinement : aspersion, recombineurs, ventilation, filtres, etc.

D’autres exigences pour ces logiciels intégraux portent sur la robustesse numérique, l'existence d'outils conviviaux d'aide à l'utilisateur pour préparer les calculs puis exploiter leurs résultats, enfin la capacité d'arrêter les calculs à un instant donné de l'accident, sauvegarder les résultats puis poursuivre les calculs jusqu'à la fin de l'accident, éventuellement avec des modifications des hypothèses (par exemple la remise en fonctionnement de systèmes de sûreté).

L'accroissement des performances des ordinateurs et l'amélioration des connaissances ont permis d'implémenter des modèles détaillés dans les logiciels intégraux précités alors que, jusqu'à la fin des années 1990, les modèles étaient aussi simples et rapides que possible, avec une grande flexibilité d'utilisation permettant des études de sensibilité. Néanmoins, malgré la sophistication progressive des modèles, les logiciels intégraux restent complémentaires des logiciels détaillés qui permettent de simuler un phénomène particulier. C'est le cas par exemple des logiciels de CFD (Computational Fluid Dynamics), utilisés par exemple pour évaluer le « risque hydrogène » dans l'enceinte de confinement ou pour traiter une explosion de vapeur (§ 5.2.2 et 5.2.3).

\subsubsection{Logiciels détaillés}

Les principaux logiciels détaillés utilisés dans le monde sont les suivants :

- pour la dégradation du cœur : ATHLET-CD (GRS en Allemagne), ICARE/CATHARE (IRSN), RELAP/SCDAPSIM (ISS aux États-Unis), SCDAP/RELAP5 (INL aux États-Unis) ;

- pour l'enceinte de confinement : COCOSYS (GRS), TONUS (IRSN), FUMO (université de Pise en Italie), GOTHIC (AECL au Canada), CONTAIN (développé par ANL aux États-Unis mais dorénavant remplacé par le logiciel intégral MELCOR) ;

- pour l'explosion de vapeur : IKEDEMO (université de Stuttgart), MC3D (IRSN) ;

- pour la mécanique des structures de grande taille : des logiciels aux éléments finis tels que ABAQUS (aux États-Unis) ou CAST3M (CEA) ;

- pour la thermohydraulique: les logiciels CFD qui résolvent les équations de Navier-Stokes dans une géométrie 3D, tels que CFX (logiciel du commerce), GASFLOW (KIT, ex-FzK en Allemagne), TONUS (IRSN).

Ces logiciels sont en général dédiés à la simulation d'une partie des scénarios d'accident ou d'une zone particulière de la centrale nucléaire, par exemple les circuits primaire et secondaire ou l'enceinte de confinement. Leur principal objectif est de réduire les incertitudes et d'aboutir à une compréhension fine des phénomènes physiques. Ils contiennent donc des modèles physiques détaillés "réalistes ॥ (dits "best-estimate") au niveau de l'état de l'art. On peut illustrer de la manière suivante la différence d'approche de ces logiciels par rapport aux logiciels intégraux : dans les logiciels détaillés une solution numérique des équations différentielles est calculée dans la plupart des cas, tandis 
que, dans les logiciels intégraux, des corrélations sont parfois utilisées, ce qui restreint leur champ d'application au domaine de validité de ces corrélations.

Les logiciels détaillés sont souvent utilisés comme références pour évaluer la validité des résultats des logiciels intégraux.

En général leur temps de calcul est élevé : avec une discrétisation très fine de l'espace et du temps, il peut falloir plusieurs semaines pour calculer un jour d'accident.

\subsection{Approche générale du développement et de la validation des logiciels}

\subsubsection{Développement des logiciels}

L'approche généralement adoptée pour le développement d'un logiciel comporte les étapes suivantes, avec des itérations possibles entre elles :

- étape 1 : définir les objectifs du logiciel, dont son champ d'application, les performances souhaitées en termes de temps de calcul, etc. ;

- étape 2 : élaborer ses spécifications générales : structure, langage de programmation, niveau de détail des modélisations, schémas numériques de résolution, etc. ;

- étape 3 : élaborer les spécifications détaillées de certaines modélisations, selon le besoin, et éventuellement réaliser des maquettes informatiques ;

- étape 4 : élaborer les modèles physiques, en général sur la base de données expérimentales ;

- étape 5 : développer le logiciel en articulant les modèles physiques avec le schéma numérique adéquat ;

- étape 6 : vérifier le logiciel en comparant sur des problèmes simples ses résultats avec des solutions analytiques, en s'assurant de la conservation de la masse et de l'énergie, de la cohérence des résultats obtenus avec divers ordinateurs, du couplage correct entre phénomènes, etc.

\subsubsection{Validation des logiciels}

Le processus de validation vise à s'assurer que le logiciel représente d'une manière fidèle la réalité des phénomènes physiques et qu'il est capable de simuler de manière fiable le déroulement complet d'un accident de fusion du cœur d'un réacteur.

Un projet du $4^{\mathrm{e}}$ PCRD (Programme cadre de recherche et de développement) de la Commission européenne, réalisé entre 1999 et 2003, a porté sur les approches de validation des logiciels : il s'agit du projet VASA (Validation Strategy of Severe Accident codes) [7], coordonné par la GRS avec un support actif de l'IRSN. Une des conclusions majeures a été de recommander une double approche : la prise en compte des phénomènes physiques mais aussi de leur importance pour la sûreté du réacteur. 
Ces deux approches sont complémentaires car la dernière permet de fixer le degré de précision attendu dans la simulation des phénomènes. Il est à noter que cette deuxième approche nécessite de modéliser tous les systèmes de sûreté des réacteurs dans les logiciels.

La validation se déroule en général en trois étapes :

- qualification d'un modèle physique implémenté dans le logiciel sur des résultats d'essais dits "à effets séparés », souvent à petite échelle, puis qualification du logiciel sur des résultats d'essais dits « à effets couplés » portant sur un ensemble de quelques phénomènes physiques, souvent réalisés avec des matériaux simulants (ces derniers essais peuvent également représenter le comportement d'un composant réel du réacteur) ;

- qualification du logiciel sur des essais intégraux, souvent réalisés à une échelle relativement grande (par exemple avec une grappe de crayons de hauteur réelle) et avec des matériaux réels, ce qui permet de vérifier l'adéquation du couplage entre les différents modèles et de vérifier qu'aucun phénomène important n'a été négligé ;

- extrapolation aux situations d'accidents pouvant affecter les réacteurs de puissance, notamment via les études des effets d'échelle, les études de sensibilité aux différents paramètres (physiques et numériques) des modèles, les comparaisons avec d'autres logiciels, et l'application à des transitoires incidentels ou accidentels réellement survenus dans des réacteurs (par exemple les accidents de TMI-2 aux États-Unis et de Fukushima Daiichi au Japon).

Lorsque ces étapes ont été réalisées de manière suffisamment complète (les progrès des connaissances nécessitent de les répéter régulièrement), il est possible de recommander aux utilisateurs des valeurs par défaut et des plages de variation pour les différents paramètres des modèles. Les conclusions de cette validation peuvent alors mettre en évidence des voies d'amélioration des modèles du logiciel.

Le domaine des accidents de fusion du cœur présente certaines particularités par rapport à d'autres domaines scientifiques : d'une part les conditions extrêmes qui interviennent tels que les températures et pression élevées, ou l'irradiation; d'autre part la très grande complexité des phénomènes. En conséquence un grand nombre de programmes expérimentaux spécifiques ont dû être menés pendant de nombreuses années dans un cadre international. Des matrices de qualification ont été bâties pour tous les logiciels en se basant sur les divers essais disponibles. Un exemple d'une matrice commune aux logiciels intégraux est présenté dans le tableau 8.1 (auquel il faut ajouter l'accident de TMI-2). Les essais retenus dans les exercices ISP (International Standard Problem) [8] de l'OECD/CSNI (Committee on the Safety of Nuclear Installations) sont largement utilisés : ces exercices consistent en des comparaisons entre logiciels sur des essais de qualité bien instrumentés. 
Tableau 8.1. Principaux programmes expérimentaux utilisés pour la qualification des logiciels intégraux.

\begin{tabular}{|c|c|c|}
\hline $\begin{array}{l}\text { Domaine ou phénomène } \\
\text { physique concerné } \\
\text { (paragraphe du livre } \\
\text { correspondant) }\end{array}$ & Nom du programme & Organisation (pays) \\
\hline \multirow{2}{*}{$\begin{array}{l}\text { Ensemble de l'accident de fusion } \\
\text { du cœur }(\S 5.1,5.5 \text { et } 7.3)\end{array}$} & LOFT-LP- FP2 & INEL (États-Unis) \\
\hline & Phébus-PF & IRSN (France) \\
\hline $\begin{array}{l}\text { Thermohydraulique du circuit } \\
\text { primaire (§ 5.1.4) }\end{array}$ & BETHSY [9] & CEA (France) \\
\hline \multirow{3}{*}{$\begin{array}{l}\text { Dégradation du cœur } \\
\text { (§ 5.1.1) }\end{array}$} & CORA & KIT (Allemagne) \\
\hline & QUENCH & KIT (Allemagne) \\
\hline & PARAMETER & LUCH (Russie) \\
\hline \multirow{2}{*}{$\begin{array}{l}\text { Relâchement des produits } \\
\text { de fission } \\
\text { (§5.5.1) }\end{array}$} & ORNL HI-VI & ORNL (Canada) \\
\hline & VERCORS & CEA (France) \\
\hline \multirow{4}{*}{$\begin{array}{l}\text { Transport des produits } \\
\text { de fission dans le circuit primaire } \\
\text { et dans l'enceinte } \\
\text { (§5.5.2) }\end{array}$} & FALCON [10] & AEAT (GB) \\
\hline & VERCORS HT & CEA (France) \\
\hline & LACE [11] & INEL (États-Unis) \\
\hline & KAEVER [12] & Battelle (Allemagne) \\
\hline \multirow{2}{*}{$\begin{array}{l}\text { Défaillance de la cuve } \\
\text { (§ 5.1.3) }\end{array}$} & LHF-OLHF & SNL (États-Unis) \\
\hline & FOREVER & KTH (Suède) \\
\hline \multirow{3}{*}{$\begin{array}{l}\text { Transferts de chaleur } \\
\text { dans un bain de corium } \\
\text { ( } § 5.4 .1 \text { et } 5.4 .2 \text { ) }\end{array}$} & COPO & VTT (Finlande) \\
\hline & ULPU [13] & UCLA (États-Unis) \\
\hline & BALI & CEA (France) \\
\hline $\begin{array}{l}\text { Fragmentation du corium } \\
\text { dans l'eau } \\
\text { (§ 5.2.3) }\end{array}$ & FARO et KROTOS & JRC Ispra \\
\hline \multirow{2}{*}{$\begin{array}{l}\text { Échauffement direct de l'enceinte } \\
(\S 5.2 .1)\end{array}$} & SURTSEY IET & SNL (États-Unis) \\
\hline & $\operatorname{DISCO}(\mathrm{C}, \mathrm{H})$ & KIT (Allemagne) \\
\hline \multirow{3}{*}{$\begin{array}{l}\text { Interaction corium-béton } \\
(\S 5.3)\end{array}$} & BETA & KIT (Allemagne) \\
\hline & $\mathrm{CCl}$ & ANL (États-Unis) \\
\hline & ACE et MACE & ANL (États-Unis) \\
\hline \multirow{3}{*}{$\begin{array}{l}\text { Chimie de l'iode dans l'enceinte } \\
(\S 5.5 .5)\end{array}$} & ACE et Phébus/RTF & AECL (Canada) \\
\hline & CAIMAN & CEA (France) \\
\hline & EPICUR & IRSN (France) \\
\hline \multirow{4}{*}{$\begin{array}{l}\text { Thermohydraulique } \\
\text { dans l'enceinte } \\
\text { (§5.2.2) }\end{array}$} & NUPEC [14] & NUPEC (Japon) \\
\hline & VANAM [14] & Battelle (Allemagne) \\
\hline & TOSQAN [15] & IRSN (France) \\
\hline & MISTRA [15] & CEA (France) \\
\hline \multirow{2}{*}{$\begin{array}{l}\text { Combustion de l'hydrogène } \\
\text { dans l'enceinte (§ } 5.2 .2 \text { ) }\end{array}$} & HDR & Battelle (Allemagne) \\
\hline & RUT & RRC-KI (Russie) \\
\hline
\end{tabular}




\subsection{ASTEC}

Le logiciel intégral ASTEC (Accident Source Term Evaluation Code) est développé depuis 1995 conjointement par l'IRSN et son homologue allemand la GRS [16]. Il joue un rôle central dans les travaux du réseau d'excellence SARNET (Severe Accident Research NETwork of excellence) des $6^{\mathrm{e}}$ et $7^{\mathrm{e}}$ PCRD de la Commission européenne, puisqu'il intègre progressivement, sous forme de modèles, l'ensemble des connaissances produites par le réseau, et que les partenaires du réseau réalisent de nombreux travaux de qualification et des comparaisons avec d'autres logiciels sur des applications aux réacteurs de puissance [17]. ASTEC est ainsi devenu le logiciel européen de référence.

\subsubsection{Capacités du logiciel}

Une première famille de versions V0 a été développée jusqu'en 2003, puis une seconde famille de versions V1 jusqu'en 2009. Mi-2009 la première version d'une troisième famille V2 a été mise en service. L'évolution majeure par rapport aux versions V1 concerne la dégradation du cœur : les modèles des versions V2, issus du logiciel détaillé ICARE2 de l'IRSN [20], sont capables de simuler la coulée du corium en 2D à l'intérieur du cœur puis vers le fond de la cuve à travers l'enveloppe et les plaques inférieures du cœur, comme cela a été observé lors de l'accident de TMI-2 (ASTEC V1 modélisait des coulées « en chandelle » en 1D le long des crayons). Une autre amélioration majeure concerne la modélisation du comportement de l'iode et du ruthénium dans les circuits et dans l'enceinte de confinement.

Ces versions V2 sont applicables aux divers types de réacteurs de génération II, REP français (900, 1300 et $1450 \mathrm{MWe})$, Konvoi allemand de 1300 MWe, REP Westinghouse de 1000 MWe et VVER de conception russe 440 ou 1000 MWe. Elles sont également applicables à des nouveaux concepts de réacteurs de génération III tels que le réacteur EPR avec son récupérateur de corium ou les réacteurs visant à conserver le corium à l'intérieur de la cuve en refroidissant cette dernière par un noyage du puits de cuve, par exemple l'AP1000 (comme l'ont montré des applications à des réacteurs VVER 440 de génération II sur lesquels ce système de refroidissement a été implanté [18]). Pour ce qui concerne les REB (réacteurs à eau bouillante), les travaux de la GRS ont montré que le logiciel ASTEC était utilisable pour ces réacteurs, hormis pour la phase de dégradation du cœur pour laquelle des adaptations de modèles sont en cours. Il en est de même pour les réacteurs CANDU et les réacteurs HTR (High Temperature Reactors) comme l'ont montré respectivement les travaux du BARC en Inde et de PBMR en Afrique du Sud.

ASTEC est également utilisable pour simuler des accidents dans les piscines de stockage de combustible, par exemple celui survenu à la centrale hongroise de Paks [19].

Le logiciel ASTEC est utilisé intensivement à l'IRSN pour les études des EPS de niveau 2 et pour des études sur les rejets envisageables en cas d'accident de fusion du cœur des REP 900 MWe et des REP 1300 MWe français. Une demande similaire est en cours pour le réacteur EPR.

La figure 8.1 montre les différents modules ainsi que la gestion des couplages entre ces modules. La grande modularité du logiciel facilite sa qualification sur des essais en n'utilisant qu'un seul module ou une suite de modules. 


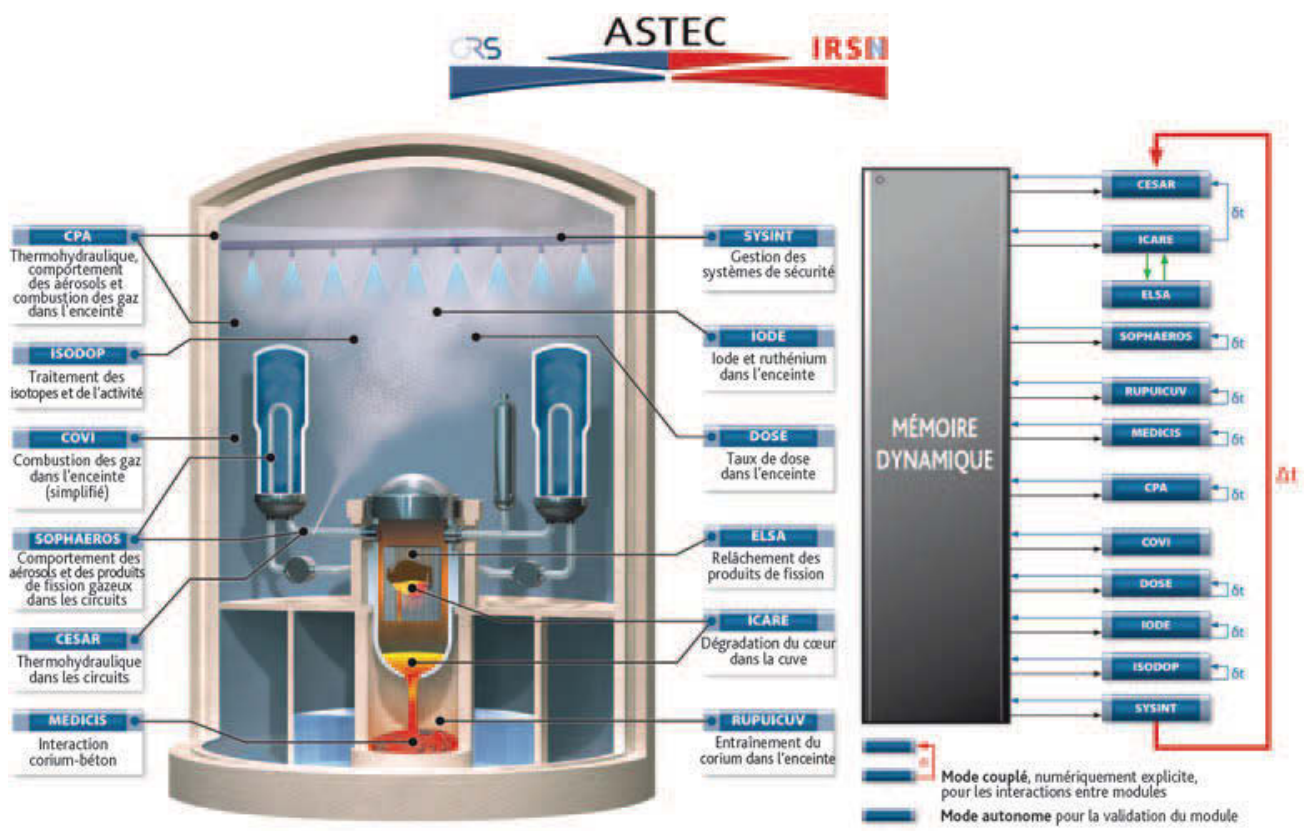

Figure 8.1. Structure d'ASTEC V2.

La version V2.0 du logiciel ASTEC a été diffusée à près de 40 organismes dans une vingtaine de pays, la plupart européens mais aussi en Russie, en Chine, en Inde, en Corée du Sud et en Afrique du Sud.

Toutes les phases d'un accident peuvent être simulées pour les cas de fonctionnement en puissance du réacteur et à l'arrêt, à l'exception des conséquences détaillées de l'entrée d'air dans la cuve (pour ce dernier aspect, des modèles figureront dans la prochaine version majeure V2.1). Tous les types de scénarios d'accidents peuvent être simulés (perte de réfrigérant primaire, rupture de tubes de générateurs de vapeur, perte des alimentations électriques, perte totale de l'alimentation en eau des générateurs de vapeur, etc.), ainsi que la plupart des procédures de conduite accidentelles telles que la dépressurisation des circuits primaire et secondaire, l'apport d'eau sur un cœur peu dégradé ou, dans l'enceinte de confinement, l'aspersion, l'éventage de l'enceinte et la filtration des rejets radioactifs. Il est également possible de représenter des dispositifs dans l'enceinte de confinement tels que les recombineurs catalytiques d'hydrogène, et, dans les VVER 440 ou les HTR, les piscines de suppression de pression par barbotage des gaz.

La thermohydraulique diphasique des circuits primaire et secondaire est modélisée par une approche numérique à cinq équations différentielles et une équation algébrique de glissement entre phases. Une approche OD à «zones » est adoptée pour décrire le comportement du corium dans le puits de cuve lors de l'interaction coriumbéton (avec une évolution possible des couches entre elles par stratification, inversion 
des couches, voir le paragraphe 5.3 pour plus de détails relatifs à l'interaction coriumbéton) et pour la thermohydraulique de l'enceinte.

L'ensemble des données de base nécessaires à un calcul est rassemblé dans une seule base (Material Data Bank) qui inclut aussi bien les propriétés physiques des matériaux et de leurs mélanges (conductivité, viscosité, etc.) que les réactions chimiques et les isotopes des produits de fission (décroissance radioactive). Les propriétés thermophysiques du corium sont issues de la base européenne de référence NUCLEA [21].

Le temps de calcul d'un scénario d'accident grave est généralement de l'ordre du temps réel sur un PC (dans un environnement Windows ou Linux). Un temps de calcul réduit à deux ou trois heures par jour d'accident est possible avec un maillage moins fin de l'espace.

Un outil (SUNSET) est mis à la disposition des utilisateurs, leur permettant de lancer automatiquement une série d'études de sensibilité pour l'analyse des incertitudes.

\subsection{2. État de la validation à mi-2013}

Le travail intensif de qualification réalisé dans les années 1990 sur les logiciels à partir desquels le logiciel ASTEC a été développé (ESCADRE, RALOC et FIPLOC) a fourni une base solide. La matrice de qualification de base contient une trentaine d'essais (issus essentiellement du tableau 8.1). Ces essais concernant les phénomènes essentiels des accidents de fusion du cœur, la matrice est appliquée à chaque livraison d'une version majeure afin de préciser les incertitudes des modèles. La qualification est complétée, pour chaque module, par des campagnes moins fréquentes mais portant sur l'ensemble des essais disponibles (ainsi par exemple une quarantaine d'essais pour le relâchement des produits de fission). Elle est également réalisée par des partenaires de l'IRSN, dans le cadre du réseau SARNET, sur des expériences de référence ayant servi de base à des exercices internationaux d'intercomparaison de logiciels de calcul (ISP) : on peut citer BETHSY 9.1b (ISP27) pour la thermohydraulique des circuits primaire et secondaire, KAEVER (ISP44) pour le comportement des aérosols dans une enceinte, et Phébus FPT1 (ISP46) pour le déroulement complet d'un accident.

Au total, l'ensemble des versions a été confronté à plus de 170 essais. Les résultats de cette confrontation sont globalement satisfaisants et démontrent que le logiciel ASTEC correspond bien à l'état de l'art en termes de compréhension et de modélisation, particulièrement pour ce qui concerne le comportement des produits de fission qui prend en compte l'ensemble des enseignements du programme expérimental Phébus-PF et des essais analytiques internationaux réalisés depuis de nombreuses années. À titre d'exemples, les figures 8.2 et 8.3 présentent respectivement les résultats de la qualification sur l'essai thermohydraulique BETHSY 9.1b (réalisé au CEA) (brèche de diamètre deux pouces dans la branche froide du circuit primaire sans fonctionnement de l'injection de sécurité à haute pression) et sur l'essai CAIMAN 97/02 (réalisé au CEA) simulant la production d'iode moléculaire et d'iode organique dans la phase gazeuse de l'enceinte de confinement au cours d'un accident grave. 


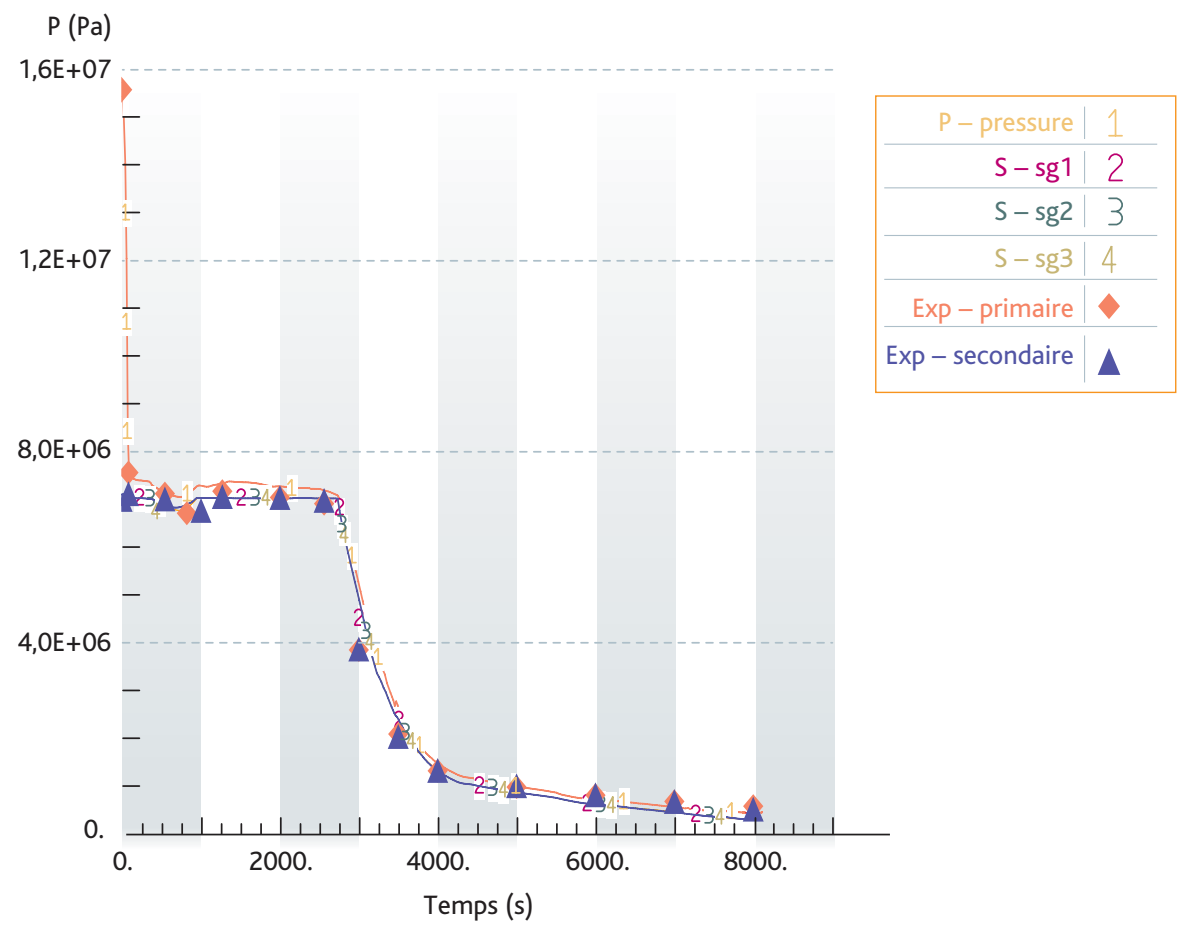

Figure 8.2. Qualification du logiciel ASTEC sur l'essai BETHSY 9.1b : évolutions des pressions primaire et secondaire calculées ; comparaison avec les valeurs expérimentales.

La priorité des améliorations actuelles des modèles du logiciel ASTEC concerne le renoyage du cœur dégradé qui est un élément essentiel pour la gestion des accidents de fusion du cœur. À l'instar de tous les autres logiciels, les modèles du logiciel ASTEC doivent être largement améliorés comme l'a montré l'interprétation des essais QUENCH et CORA, et surtout l'incapacité à reproduire les pics de production d'hydrogène observés lors de ces essais de renoyage.

Au-delà des comparaisons aux résultats expérimentaux, il faut démontrer la capacité du logiciel à calculer l'ensemble des accidents graves pouvant affecter les réacteurs de puissance. ASTEC a ainsi été utilisé pour les études de l'EPS2 REP 1300 de l'IRSN relatives aux principales familles de scénarios (brèche dans le circuit primaire ou le circuit secondaire, perte des alimentations électriques, etc.) avec des variantes examinant le fonctionnement ou non des systèmes de sauvegarde du réacteur. Près d'une centaine de calculs de séquences ont été effectués, ce qui a permis de vérifier que les résultats obtenus étaient cohérents entre eux et que les tendances obtenues étaient physiquement crédibles.

Des comparaisons ont également été réalisées avec d'autres logiciels sur certains scénarios d'accident. Ainsi, dans le cadre du réseau SARNET, des comparaisons ont été 


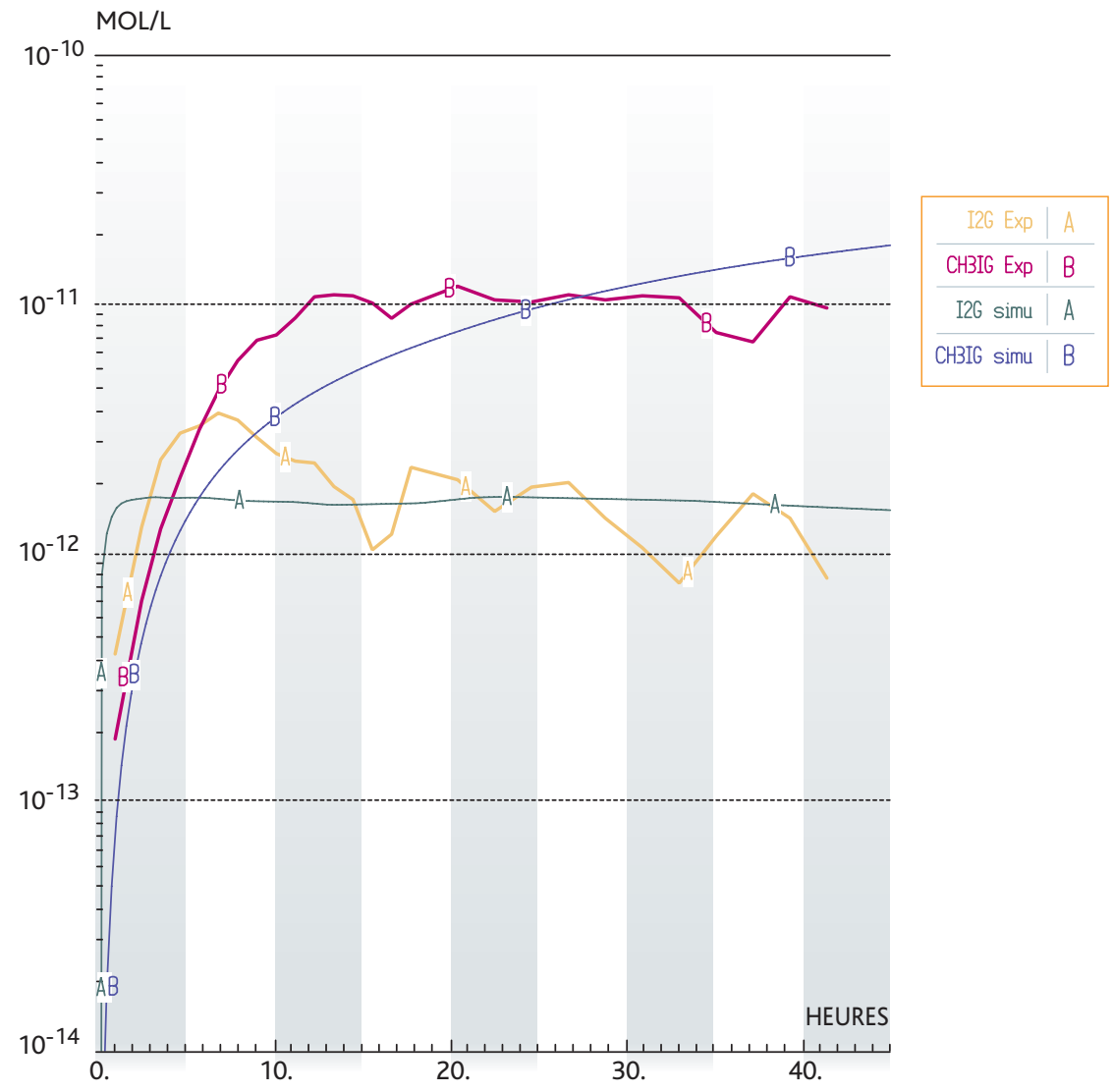

Figure 8.3. Qualification du logiciel ASTEC sur l'essai CAIMAN 97/02 : évolutions des concentrations d'iode dans la phase gazeuse de l'enceinte sous forme d'iode moléculaire $\mathrm{I}_{2}$ et sous forme d'iode organique $\mathrm{CH}_{3}$.

effectuées avec les logiciels intégraux MELCOR et MAAP4 et avec des logiciels détaillés tels que ICARE/CATHARE et CONTAIN, pour des réacteurs de divers types (REP 900, REP 1300 , Konvoi 1300, VVER 440 et VVER 1000). Globalement les résultats ont été jugés proches mais des écarts sont observés lors de certaines phases des scénarios. Ces comparaisons ont permis d'identifier les modèles à l'origine de ces écarts et contribué ainsi à la quantification des incertitudes sur l'évaluation de certains phénomènes physiques.

\subsubsection{Perspectives d'évolution du logiciel ASTEC}

Dans la prochaine version majeure V2.1 du logiciel ASTEC qui devrait être disponible en 2014, plusieurs nouveaux modèles seront intégrés résultant de l'interprétation des 
programmes expérimentaux lancés en vue de réduire les incertitudes sur les connaissances, par exemple :

- pour la chimie du transport de l'iode et du ruthénium dans le circuit primaire : les essais CHIP de l'IRSN (§ 5.5.6.2) réalisés dans le cadre du programme ISTP (International Source Term Program) qui permettent de qualifier un nouveau modèle de cinétique chimique ;

- pour la chimie de l'iode et du ruthénium dans l'enceinte de confinement: les essais EPICUR de l'IRSN (§ 5.5.6.2), réalisés dans le cadre du programme ISTP ;

- pour la distribution spatiale du flux de chaleur dans le bain de corium au cours de l'interaction corium-béton : les essais VULCANO et CLARA du CEA, et les essais du projet $\mathrm{CCl}$ avec des matériaux réels ;

- pour l'effet d'un taux de combustion élevé du combustible et l'effet du combustible MOX sur la dégradation du cœur et sur le relâchement des produits de fission : les essais VERCORS puis VERDON du CEA ;

- pour le renoyage d'un cœur dégradé : les essais PEARL de l'IRSN de refroidissement d'un lit de débris (et les travaux associés prévus par les partenaires du réseau SARNET).

En parallèle se déroulent des travaux d'adaptation des modèles de dégradation du cœur aux REB en collaboration avec la GRS et l'université de Stuttgart et aux réacteurs CANDU en collaboration avec le BARC.

Le logiciel ASTEC devrait garder dans la poursuite du réseau SARNET son rôle de capitalisation des connaissances sur les accidents graves. Des efforts sont conduits pour accélérer les calculs, notamment via la parallélisation.

Depuis 2009, des travaux portent sur l'adaptation d'ASTEC aux accidents dans les réacteurs de génération IV à neutrons rapides refroidis au sodium et dans les installations de fusion comme ITER.

\subsection{MAAP}

Le développement du logiciel MAAP (Modular Accident Analysis Program) a débuté aux États-Unis au début des années 1980 pour servir aux études physiques en support des EPS dans le cadre du programme de recherche IDCOR (Industry Degraded Core Rulemaking) qui regroupait une soixantaine d'industriels américains. Depuis l'arrêt du programme IDCOR, l'EPRI (Electric Power Research Institute, organisme de R\&D américain) est propriétaire du logiciel MAAP ; les développements sont toujours réalisés par la société Fauske \& Associates, Inc (FAI).

De nombreux exploitants nucléaires ont acquis une licence d'utilisation du logiciel MAAP et l'utilisent pour leurs études de sûreté. Ils sont regroupés au sein d'un groupe d'utilisateurs comportant plus de 55 organisations (le MAAP Users Group).

Le logiciel MAAP est utilisé par EDF pour étudier les accidents de fusion du cœur des REP : EPS de niveau 2, dimensionnement des recombineurs d'hydrogène, réévaluation 
des rejets envisageables en cas d'accident de fusion du cœur, études en support à l'élaboration du guide d'intervention en cas d'accident grave (GIAG), étude du risque d'échauffement direct de l'enceinte $(D C H)$, étude de la montée en pression lente de l'enceinte, etc.

Depuis son acquisition du logiciel en 1991, EDF a développé une compétence propre en termes de développement et de validation du logiciel MAAP en s'appuyant notamment sur la réalisation de comparaisons des résultats fournis par différents logiciels pour des applications à des réacteurs de puissance.

EDF a continué dans cette logique de développement en réalisant à partir de 1996 des versions propres du logiciel MAAP contenant des apports spécifiques. La version en exploitation du logiciel MAAP à EDF est la version 4.07a qui permet de modéliser des séquences accidentelles des réacteurs EPR en complément de celles des REP 900 et $1300 \mathrm{MWe}$.

\subsubsection{Capacités du logiciel}

Le logiciel MAAP permet de traiter les situations accidentelles d'un réacteur REP (dont les réacteurs VVER et EPR), REB ou CANDU (mais avec des versions différentes du logiciel pour chacun de ces types de réacteurs), en particulier les séquences avec fusion du cœur, quel que soit l'état initial de l'installation (en puissance ou à l'arrêt).

La modélisation fonctionnelle est prévue pour pouvoir examiner l'effet d'actions des opérateurs sur le déroulement des séquences accidentelles.

Son temps de calcul est réduit : environ deux heures de calcul sur un PC (dans un environnement Linux) pour simuler 24 heures de temps réel pour une séquence avec dégradation du cœur.

La modélisation du transport des produits de fission (relâchements lors de la dégradation du cœur, migration dans le circuit primaire et dans l'enceinte, chimie) permet de déterminer les rejets dans l'environnement ainsi que les contaminations surfaciques et volumiques dans les locaux.

Le logiciel MAAP résout, pour chaque volume de contrôle, les équations de conservation de la masse et de l'énergie. Les équations de conservation de la quantité de mouvement ne sont pas des équations différentielles et se résument à des équations de Bernoulli.

Hors pressuriseur, le circuit primaire est représenté par 14 volumes au maximum et l'enceinte est modélisée par 30 volumes au maximum. Le cœur est modélisé de façon axisymétrique avec 175 mailles au maximum.

À titre d'illustration, la figure 8.4 montre les principaux phénomènes physiques modélisés par le logiciel MAAP pour le circuit primaire et l'enceinte de confinement. 


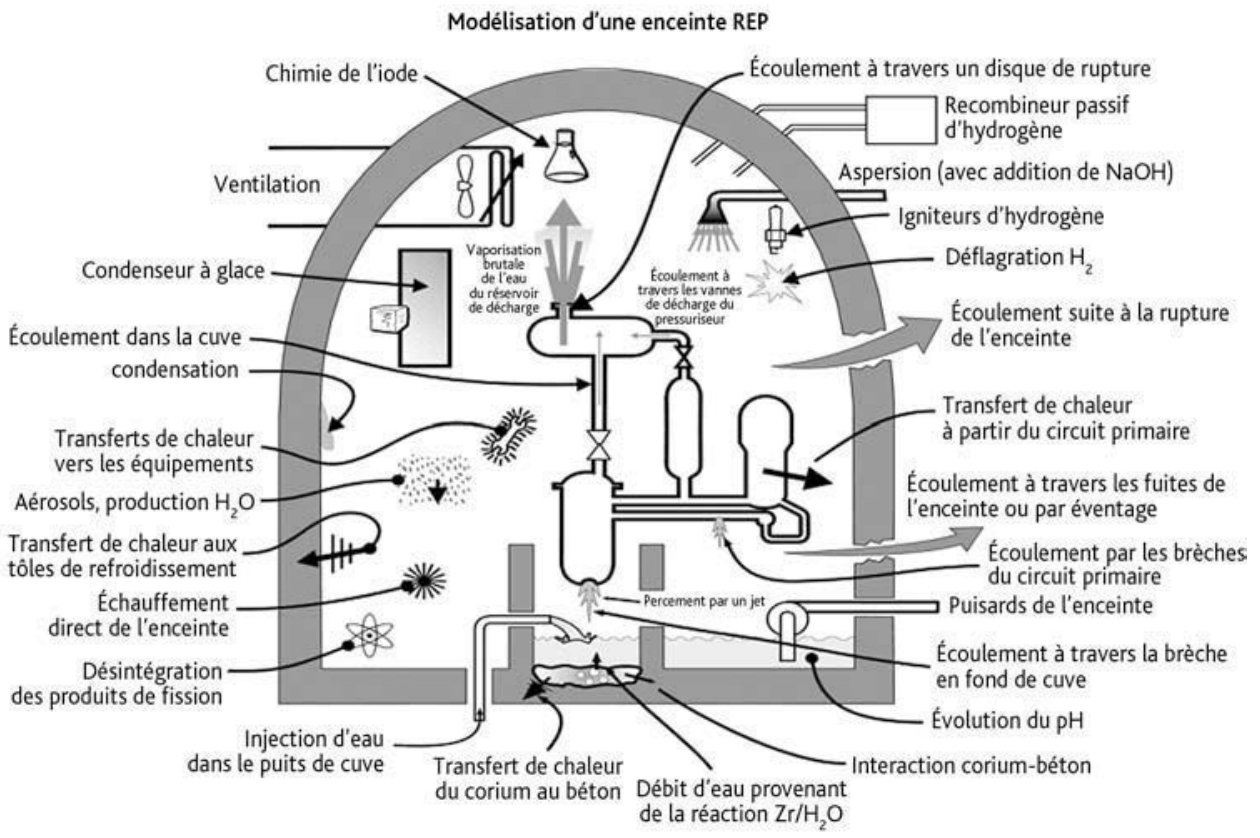

Figure 8.4. Modèles du logiciel MAAP pour les phénomènes physiques dans l'enceinte d'un REP.

La version MAAP4.07c comporte des modèles spécifiques adaptés aux équipements du réacteur EPR [22] ; on peut citer :

- la modélisation du réflecteur lourd en périphérie du cœur : le logiciel modélise la formation d'une croûte entre le corium fondu et le réflecteur lourd, la formation d'une couche d'acier fondu entre la croûte et la région solide du réflecteur lourd, la percée du réflecteur lourd puis l'ablation de la paroi externe du réflecteur lors de l'écoulement du corium dans l'espace annulaire situé entre le réflecteur et la cuve (figure 8.5) ;

- la modélisation du rayonnement du bain de corium dans le puits de cuve vers les structures de la cuve et les parois du puits de cuve avant la fusion de la trappe inférieure ;

- la prise en compte de la diversité des bétons et des configurations des parois du compartimentage de l'enceinte et de la chambre d'étalement pour le calcul de l'interaction corium-béton.

Une nouvelle version du logiciel MAAP4 (développée par EDF R\&D sur la base de la version EPRI standard) a été mise en exploitation par EDF en 2012. Cette version inclut les dernières avancées issues des projets internationaux (SARNET, ISTP, etc.) notamment pour ce qui concerne la réduction des incertitudes sur la dégradation du cœur et l'évaluation des rejets. Les améliorations des modèles portent par exemple sur l'oxydation par l'air des gaines des crayons combustibles, l'oxydation du $\mathrm{B}_{4} \mathrm{C}$, les relâchements 


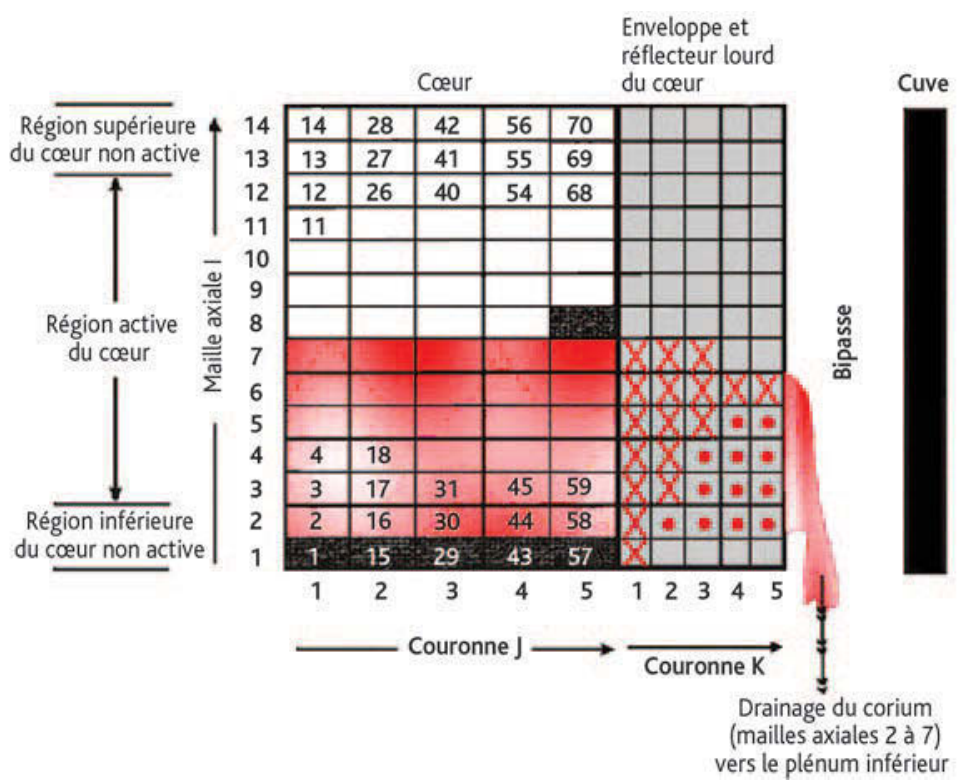

Figure 8.5. Modélisation de l'ablation du réflecteur lourd du réacteur EPR au cours de la dégradation du cœur.

de produits de fission peu volatils et semi-volatils depuis le combustible, la chimie de l'iode dans l'enceinte, le traitement de l'interaction corium-béton et un couplage par tabulation entre le logiciel MAAP et la base NUCLEA afin d'améliorer l'évaluation des propriétés thermophysiques du corium.

\subsection{2. État de la validation à mi-2013}

Dans la pratique, la validation physique du logiciel MAAP est un processus continu qui s'alimente de la simulation de nouveaux essais. Ainsi, EDF contribue à augmenter régulièrement la base de qualification du logiciel MAAP, en participant notamment aux ISP et à des projets internationaux (SARNET, ISTP, etc.).

En plus des nombreux tests réalisés par FAI, EDF a, depuis son acquisition du logiciel, réalisé des calculs complémentaires sur des essais, en particulier sur la dégradation du cœur, la production d'hydrogène, la thermohydraulique dans l'enceinte, la chimie de l'iode, les relâchements de produits de fission, la tenue mécanique de la cuve. Dans l'ensemble, la grille de qualification du logiciel MAAP4.07c couvre les expériences des programmes répertoriés dans le tableau 8.1.

Le logiciel est également qualifié par EDF sur les essais intégraux disponibles (Phébus, LOFT) et sur l'accident de TMI-2 en complément de la qualification menée sur des essais analytiques ou à effets séparés.

Le logiciel MAAP est également comparé à d'autres logiciels, parmi lesquels des logiciels mécanistes tels que ASTER (comportement mécanique de la cuve), RELAP ou RETRAN (logiciels américains sur la thermohydraulique du circuit primaire), ou des 
logiciels intégraux comme ASTEC. Des comparaisons avec le logiciel ASTEC ont été réalisées dans le cadre du réseau SARNET pour les REP $900 \mathrm{MWe}$ : il a été constaté que les tendances d'évolution des principaux résultats, ainsi que leurs ordres de grandeur, étaient proches pour les logiciels ASTEC et MAAP appliqués à diverses séquences accidentelles avec des brèches primaires de taille et de localisation différentes.

\subsubsection{Perspectives d'évolution du logiciel MAAP}

La perspective est le passage d'EDF à la version MAAP 5.0 envisagée début 2015 . Cette version correspond à une évolution de grande ampleur du logiciel. Elle intègre la majorité des modèles physiques développés par EDF dans ses propres versions de MAAP 4 (dégradation des barres de contrôle en $\mathrm{AIC}$ ou $\mathrm{B}_{4} \mathrm{C}$, oxydation du zirconium à haute température, relâchements de produits de fission depuis la matrice du combustible, etc.). Elle inclut également de nombreuses améliorations de modèles physiques développées par FAI dont certaines sont détaillées ci-dessous :

- thermohydraulique dans le circuit primaire: mise en place d'un traitement beaucoup plus détaillé avec découpage du circuit primaire en 49 mailles, traitement d'un fluide monophasique ou diphasique eau-vapeur, possibilité de simuler l'injection de sécurité dans la branche froide seulement, amélioration de la modélisation de la circulation naturelle dans la cuve (notamment entre l'espace annulaire, le cœur et le plénum supérieur), amélioration de la modélisation de la formation de bouchons d'eau et de leur influence sur la circulation naturelle, prise en compte de l'inversion éventuelle de débit dans les tubes des générateurs de vapeur);

- modélisation des accumulateurs: modélisation des accumulateurs en tant que région du circuit primaire, modélisation de la paroi des accumulateurs en tant que puits de chaleur, injection d'azote dans le circuit primaire depuis les accumulateurs ;

- modélisation du cœur : introduction de modèles de neutronique 1D et cinétique point ;

- modélisation de la rétention du corium dans la cuve : raffinement du maillage axial de la cuve jusqu'à 100 mailles, modélisation de la variation du flux critique d'assèchement le long de la face externe de la cuve (en fonction de son angle d'inclinaison) lorsque celle-ci est supposée noyée, calcul des transferts thermiques par ébullition nucléée entre la surface externe de la cuve et l'eau liquide introduite dans le puits de cuve ; modélisation de la présence d'un calorifuge et d'un jeu entre la cuve et le calorifuge ;

- nouvelles modélisations de phénomènes intervenant dans l'évaluation des transferts de chaleur dans l'enceinte : convection naturelle et convection forcée dans les compartiments de l'enceinte induites par le fluide sortant d'une brèche dans le circuit primaire, condensation sur les parois en tenant compte des peintures, entraînement par les gaz des gouttelettes d'eau condensées sur la paroi, condensation d'un jet de vapeur dans un compartiment noyé, combustion d'hydrogène. 
Ces évolutions ont été financées par une quinzaine d'organisations qui possèdent la licence d'utilisation du logiciel MAAP4. Après l'amélioration de la modélisation physique, la société FAI a concentré ses efforts en 2011-2012 sur la stabilité numérique du logiciel MAAP5. À partir de 2015, la nouvelle version MAAP5 pourrait devenir une alternative à MAAP4 pour les études réalisées par EDF dans le domaine des accidents de fusion du cœur.

\subsection{MELCOR}

MELCOR est un logiciel intégral développé depuis 1982 par SNL à la demande de l'US NRC pour permettre l'étude complète d'un accident de fusion du cœur pouvant affecter un réacteur à eau légère (REP y compris les VVER, REB) [23]. Des applications ont été faites également pour des réacteurs RBMK, ainsi que, de manière très exploratoire, pour des réacteurs CANDU. Des partenaires étrangers contribuent à la validation du logiciel dans le cadre du Cooperative Severe Accident Research Program (CSARP) de l'US NRC.

Le logiciel est utilisé par l'US NRC pour les réévaluations des rejets radioactifs, par exemple pour les combustibles MOX ou les combustibles à haut taux de combustion, pour l'évaluation de nouveaux concepts de réacteurs (AP1000 de Westinghouse, ESBWR, US.EPR, etc.), pour l'estimation réaliste des conséquences d'accidents de fusion du cœur pour les différents réacteurs exploités aux États-Unis en tenant compte des connaissances, méthodes et logiciels les plus récents et des incertitudes associées, et pour l'étude d'accidents dans les piscines de stockage de combustibles usés. Au niveau international, les applications concernent en particulier l'optimisation des guides de gestion des accidents.

Le logiciel MELCOR a remplacé l'ensemble des logiciels développés pour l'US NRC dans les années 1980 aux États-Unis et prend en compte la quasi-totalité des phénomènes physiques mis en jeu lors d'un accident, comme le montre la figure 8.6.

La version la plus utilisée en 2013 est la version MELCOR 1.8.6, livrée en 2005. Les améliorations de modèles réalisées à cette époque concernaient principalement :

- la formation et l'évolution d'un bain de corium dans la cuve ;

- le relâchement de l'argent et l'oxydation du $\mathrm{B}_{4} \mathrm{C}$ des barres de commande ;

- les relâchements de produits de fission à partir des combustibles MOX et des combustibles à hauts taux de combustion ;

- l'introduction d'un modèle de cinétique point pour calculer les évolutions de la réactivité.

Une particularité du logiciel MELCOR (par rapport aux logiciels ASTEC et MAAP) est l'unicité des modèles thermohydrauliques utilisés pour traiter toutes les zones du réacteur, tant pour le circuit primaire et le circuit secondaire que pour l'enceinte de confinement. Une approche numérique à cinq équations différentielles et une équation algébrique de glissement entre phases est utilisée. La méthode de résolution 


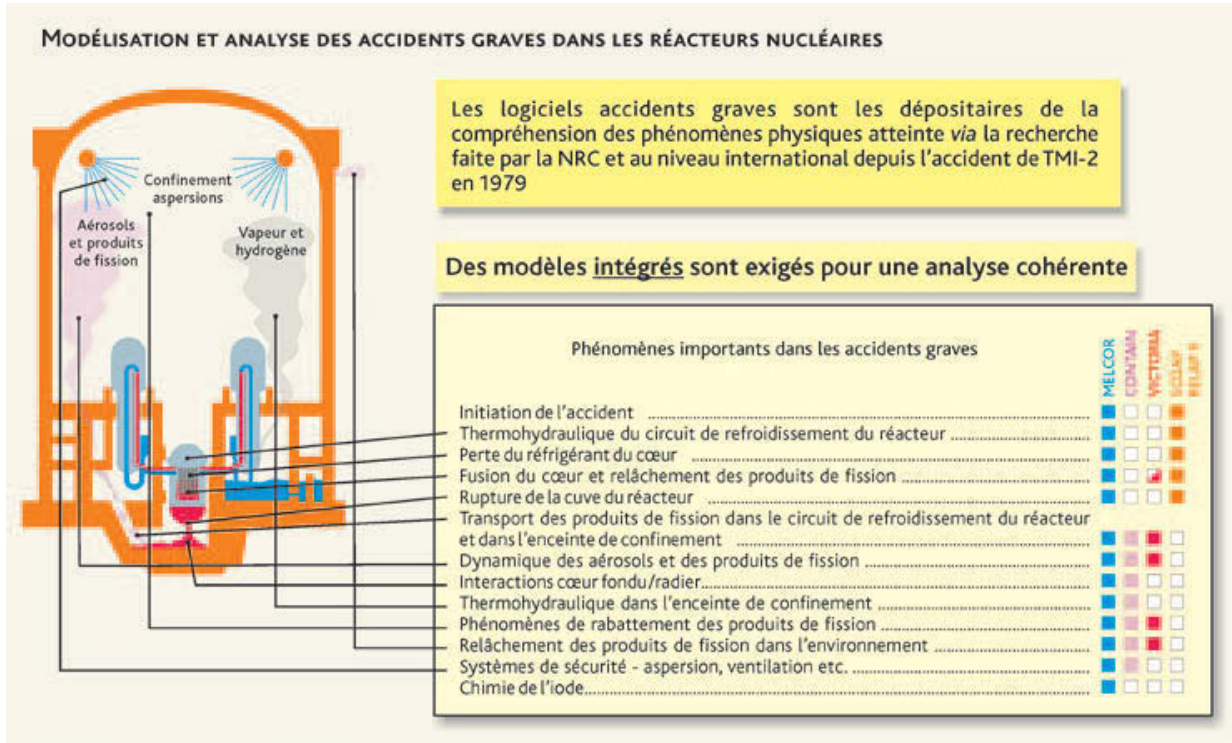

Figure 8.6. Phénomènes physiques modélisés par le logiciel MELCOR.

semi-implicite en temps des équations de bilan limite la valeur du pas de temps de calcul afin d'assurer la stabilité du schéma numérique.

Un outil est mis à la disposition des utilisateurs, qui permet de lancer automatiquement une série d'études de sensibilité pour l'analyse des incertitudes.

Une illustration d'un résultat de calcul a posteriori de l'essai Phébus FPT1 (réalisé par l'IRSN) est présentée sur la figure 8.7. Cet essai concernait le début de la dégradation d'une grappe de crayons REP (oxydation des gaines, dissolution de l'UO ${ }_{2}$, coulées de corium, etc.). La figure 8.7 montre une comparaison entre calcul et mesure de la masse d'hydrogène relâché au cours de l'essai.

Le travail de qualification des modèles relatifs à la chimie de l'iode, notamment sur la base des résultats des essais Phébus-PF, a mis en évidence la nécessité d'améliorer significativement certains de ces modèles, en particulier ceux influant sur la concentration calculée de l'iode en phase gazeuse dans l'enceinte de confinement.

Une nouvelle série de versions (MELCOR 2) est en cours de développement (la version 1.8.6 ne fait plus l'objet que d'une maintenance corrective). La version 2.1 a été diffusée en septembre 2009. La structure informatique du logiciel a été modernisée (par exemple passage au langage de programmation Fortran 95) et les jeux de données restructurés. Des efforts considérables ont été faits en matière d'assurance de la qualité (tests de non-régression systématiques, etc.), de support aux utilisateurs avec la fourniture d'un outil d'aide « en ligne » accessible via le web, et d'accroissement de la convivialité des outils de pré et post-traitement. 


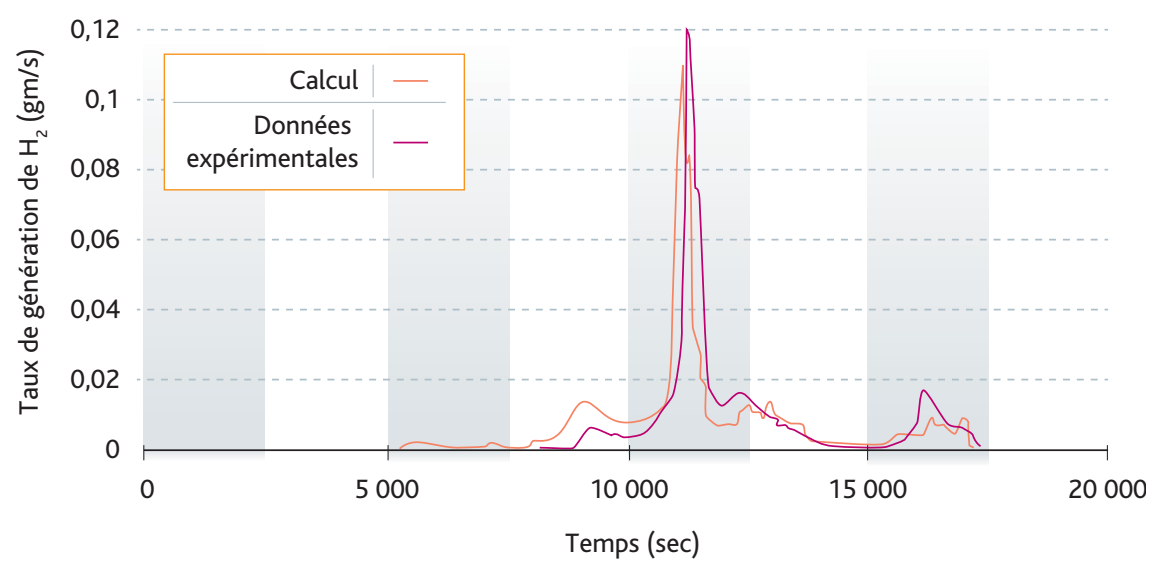

Figure 8.7. Comparaison entre mesures et calculs MELCOR 1.8.6 du débit de relâchement d'hydrogène pendant l'essai Phébus FPT1.

En 2010-2011, les travaux de modélisation portaient sur :

- les réacteurs de génération III, REP (US EPR, AP1000, etc.) ou REB (ABWR, etc.). La modélisation de certains phénomènes physiques a été améliorée sur la base des avancées des connaissances, par exemple sur le comportement de l'iode en phase gazeuse dans l'enceinte de confinement ;

- le comportement du combustible $\mathrm{UO}_{2}$ à forts taux de combustion et du combustible MOX ;

- les réacteurs de génération IV, incluant une généralisation des fluides traités (sodium, sels fondus, etc.). Les efforts portent principalement sur les réacteurs HTR, à boulets ou à prismes, pour lesquels des modèles de transfert de chaleur à l'intérieur des boulets ou avec l'hélium, d'oxydation du graphite, de relâchement des produits de fission à partir du combustible, de génération et de transport de poussières sont dévelopés.

\section{Références}

[1] N. Rasmussen, Rapport WASH 1400, The reactor safety study, 1975.

[2] NUREG, 1150, Severe Accident Risks: An assessment for five US Nuclear Power Plants, 1991.

[3] M. Kajimoto et al., Development of THALES2, a Computer Code for Coupled Thermal-Hydraulics and Fission Product Transport Analysis for Severe Accidents at LWRs and its Application to Analysis of Fission Product Revaporization Phenomena, Proceedings of the ANS International Topical meeting on safety of thermal Nuclear reactors, Portland (États-Unis), juillet 1991. 
[4] B. Linet, A. Maillat, ESCADRE code development and validation - An overview, Proceedings of the International Topical meeting Severe Accident Risk and management (SARM'97), Piestany (Slovakia), juin 1997.

[5] M. Naitoh, S. Hosoda, C.M. Allison, Assessment of water injection as severe accident management using SAMPSON code, Conference ICONE-13, Beijing, China, 16-20 mai 2005.

[6] L.A. Bolshov, V.F. Strizhov, SOCRAT - The System of Codes for Realistic Analysis of Severe Accidents, Conférence ICAPP '06, Reno (États-Unis), juin 2006.

[7] H.-J. Allelein et al., Validation strategies for severe accident codes (VASA), EU Cosponsored research on Containment Integrity, EUR 19952 EN, Brussels, p. 295$324,2000$.

[8] CSNI: International Standard Problems (ISP), brief descriptions (1975-1999), NEA/CSNI/R(2000)5, 2000.

[9] CSNI integral test facility validation matrix for the assessment of thermal-hydraulic codes for LWR LOCA and transients, NEA/CSNI/R(96)-17, 1996.

[10] A.M. Beard, P.J. Bennett, B.R. Bowsher, J. Brunning, The Falcon Programme: Characterisation of Multicomponent Aerosols in Severe Nuclear Reactors Accidents, Journal of Aerosol Science 23, S831, 1992.

[11] F. Rahn, R. Sher, R.C. Vogel, Summary of the LWR aerosol containment experiments (LACE) programme, IAEA Symposium Severe Accidents in Nuclear Power Plants, Sorrento, 21-25 mars 1988, ISBN 92-0-020188-1.

[12] M. Firnhaber, K. Fischer, S. Schwarz, G. Weber, ISP-44 KAEVER tests - Experiments on the behaviour of core-melt aerosols in a LWR containment, NEA/CSNI/R(2003)5, 2003.

[13] Theofanous and Syri, The coolability limits of a reactor pressure vessel lower head, Nuclear Engineering and Design 169 (1-3), 59-76, 1997.

[14] SOAR on Containment Thermalhydraulics and Hydrogen distribution, NEA/ CSNI/R(99)-16, 1999.

[15] H.J. Allelein et al., International standard problem ISP-47 on containment thermal-hydraulics, Final report, NEA/CSNI/R(2007)10, 2007.

[16] J.P. Van Dorsselaere, C. Seropian, P. Chatelard, F. Jacq, J. Fleurot, P. Giordano, N. Reinke, B. Schwinges, H.J. Allelein, W. Luther, The ASTEC integral Code for severe accident simulation, Journal Nuclear Technology 165, 293-307, 2009.

[17] T. Albiol, J.P. Van Dorsselaere, N. Reinke, SARNET: a success story. Survey of major achievements on severe accidents and of knowledge capitalization within the ASTEC code, Conférence EUROSAFE, Paris, Novembre 2008. 
[18] D. Tarabelli, G. Ratel, P. Pélisson, G. Guillard, M. Barnak, P. Matejovic, ASTEC application to in-vessel corium retention, Nuclear Engineering and Design 239, 1345$1353,2009$.

[19] J. Elter, P. Matejovic, Proposal of in-vessel corium retention concept for PAKS NPP, OECD MASCA2 Seminar, Cadarache (France), 11-12 octobre, 2007.

[20] P. Chatelard, J. Fleurot, O. Marchand, P. Drai, Assessment of ICARE/CATHARE V1 severe accident code, Conférence ICONE-14, Miami, Florida (États-Unis), 17-20 juillet, 2006.

[21] B. Cheynet, P. Chaud, P.Y. Chevalier, E. Fischer, P. Mason, M. Mignanelli, NUCLEA: Thermodynamic Properties and Phase Equilibria in the Nuclear Systems of Interest, Journal de Physique IV 113, 61-64, 2004.

[22] E. Williams, R. Martin, P. Gandrille, R. Meireles, R. Prior, C. Henry, Q. Zhou, Recent revisions to MAAP4 for US EPR Severe Accident Applications, Conference ICAPP 08, Anaheim (CA, États-Unis), juin 2008.

[23] R.O. Gauntt et al., MELCOR Computer Code Manuals, Version 1.8.6, Rapport SAND 2005-5713, 2005. 
